\title{
Research on Computer Network Remote Control Technology
}

\author{
Gang Chen, Dawei Zhao \\ College of Humanities \& Sciences of Northeast Normal University, Changchun, Jilin, 130117
}

Keywords: Computer, Network, Remote Control Technology

\begin{abstract}
With the development of science and technology, the technology of science and technology has been obviously improved. Computer network technology has also been widely used and popularized. The application of computer network remote control technology in various industries can greatly reduce the management cost of each unit, reduce energy consumption, and effectively improve the work efficiency in all aspects which is one of the directions that industries must attach great importance to in the future. Among people's daily life, computer network remote control system has become a key application technology among computer application systems, whose importance is self-evident. This paper mainly analyzes the computer network remote control system, and takes the distance education system as an example, analyzes the design and application of the system.
\end{abstract}

\section{Introduction}

Under normal circumstances, automatic control mainly refers to the case of no one under control, the device in accordance with the predetermined procedures for self-operation, the continuous development and application of computer technology in the process of computer network remote control technology can be better achieved System of self-control and regulation, to ensure the stability and reliability of the entire system has a significant impact. Therefore, in the computer control system to play a powerful data collection, transmission, application and other functions under the circumstances, the entire system can be more efficient and safe operation, is an important guarantee for the realization of automatic control system. It can be seen from this that in the era of information networks, the combination of computers and intelligent control systems is an inevitable trend for the continuous development of society and plays an important role in promoting the sustainable development of mankind and economy. In practice, the computer remote control system includes computer hardware control, computer software control two aspects, of which computer software to ensure that the system runs in accordance with the set program is to reduce the accidental safety accidents is an important foundation, which is mainly composed of computer Language compiled from, and computer hardware, including control objects, computers, related equipment and channels, etc., with many types, functions, to protect the performance of automatic control system has a tremendous role. At present, the computer network remote control technology is more commonly used system supervisory control system, operation guidance control system, direct digital control system and field bus control system, and people's lives, work and study and other aspects are very closely linked, is China's information technology, network technology, the future development needs one of the directions attached great importance.

\section{Basic Concepts and Principles of Computer Communication and Network Remote Control Technology}

Computer communication technology and computer network remote control technology there is a certain relationship between the two. Computer network remote control technology is through the use of Internet technology, the use of related remote control software to achieve mutual communication and exchange between computers, remote control software is mainly composed of both client software and server-side program, the same remote control the software is installed on two computers that need to communicate. Through the network, two computers can communicate 
with each other through the software. The process is mainly the result of the computer's console and server working together. The server can effectively sort and sort the collected information. On this basis, the controller compiles and sends the processed information preparation, at the same time, but also to achieve the supervision and control of information transmission.

Its working principle is mainly the method of using manual operation to quickly and safely transfer information on one computer to another computer, and on this basis, it can also realize the basic control and adjustment of the computer. By using Remote control software and the formation of two computers, there is a certain degree of relevance of network data, this data is called IP protocol, IP protocol is an important basis for the interconnection between computers, you must have the IP protocol to ensure that two computers can connect to the network and work properly. IP protocol can be subdivided into UDP protocol and TCP protocol. There are obvious differences and differences in functions between the two protocols. UDP data transmission mainly organizes, decomposes and transmits the collected data. TCP protocol is mainly through the computer to establish the appropriate data stream to meet the computer information exchange process required by the data support. In the process of computer connection, in order to effectively ensure the accuracy of information transfer, TCP protocol has better protection in data analysis and transfer, mainly because TCP protocol can fully connect the server terminal and the computer Relationship, to ensure the stability and accuracy of the information transfer process.

\section{Components of Computer Network Remote Control System}

Remote control technology mainly means that computers in different networks are interconnected through remote dialing, Internet access or computer network, and thus remote computer system maintenance, remote guidance and management control are realized. Want to carry on the computer long-range control, first needs many computers to enter together to have a certain available network among them, this network may be any network, like World Wide Web, the local area network. In addition, there is a need for some software that can be remotely controlled using a PC's LPT or COM port via cable. Computer remote control software is mainly by the controlled terminal program Server and Client Control two parts. In the actual use process, the two software programs must be in normal operation, through the service agreement, so that a variety of remote control functions will control commands transmitted from the control side to the controlled end, and thus achieve the purpose of remote control.

Computer network remote control system mainly consists of 3 parts. (1) Master network. In general, the main control network refers to the network program that adjusts and controls the network system, which can improve and handle the internal communication, evaluation and external connection of the computer network. The main body of this part is information, based on the network coordination function, perfect to meet people's daily needs. Master network is mainly to provide remote control services, to meet the more complex structure of the network, to achieve mutual coordination of information delivery, in order to achieve remote control of the computer network. (2) Communication protocol. Communication protocol ensures data security, reliable transmission. With the rapid development of network technology, the development of communication protocols has become a very important indicator. Under normal circumstances, the communication protocol includes PTP protocol, RTCP protocol, TCP protocol and IP protocol. The content of the protocol mainly includes notification return, byte communication, serial number application and connection oriented. Relying on communication protocols, remote control systems can be more suitable for a variety of network environments. (3) Controlled network. Controlled networks enable networked control as a foundation, advanced services based on advanced networking technology, and the use of high-quality hardware and high-performance software programs to provide better service to users with a high degree of stability and security.

\section{Applications of Computer Network Remote Control System}

Computer network remote control technology at work and the actual life of the applications are 
very extensive, such as remote monitoring, distance education and remote office and so on.

For network system renovation and computer maintenance, engineers and related managers are generally required to visit the site for maintenance and renovation. However, the use of remote control system, you do not need engineers or related managers to visit the scene, only need to be on the network directly to the scene to install, configure, maintain and manage, such remote management tools to greatly reduce the maintenance staff Time, but also directly reduce the user's loss, improve the efficiency of maintenance staff, so that both are more convenient. With the development of science and technology, many scientific and technological elements have also been incorporated into education. They are not only an educational environment, but also an effective improvement in the quality of education and educational resources. Educational methods are constantly being innovated as science and technology advance. From the initial multimedia teaching to the most advanced distance education at present, this shows the progress of science and technology in education. Distance education can provide students with more educational opportunities. Although students cannot face teachers in real face, they can still be directly taught and counseled by teachers. In addition, distance learning can also improve students 'learning enthusiasm and teachers can also directly view the students' problem-solving ideas and learning styles so as to make education better.

The use of remote control system to achieve the remote office, allowing office workers in any network wherever they are able to office, let the office automation on the Internet for the purpose. Make the office realize anytime, anywhere, anywhere can be involved in the office of the computer which can download resources at any time, access to files, watch computer hard drive which all kinds of data and information. Currently in many developed countries, this technology has been relatively mature. Telecommuting not only can greatly improve the work enthusiasm and work efficiency, but also can enhance the understanding and communication among staff. In addition, telecommuting technology can help society mitigate traffic pressure and environmental pollution.

In some industries, computer network remote control system can also play a significant role. For example, the coal mining industry, which uses the computer network remote control system reasonably in this industry, can realize timely safety early warning and ensure its long-term safe working status. At the same time, the system enables timely safety warnings and rapid response to disasters. The application of remote control system in the coal mining industry can effectively promote the efficient production of coal mining industry, provide security for safe operation and reduce property loss. Reasonable use of remote control system can effectively reduce the security risks due to human factors, to ensure the stable operation of coal mining industry.

\section{Conclusions}

With the progress of science and technology, new computer remote control technology emerges one after another, bringing more conveniences and innovations to people's daily life. According to the reasonable application of computer network remote control technology, the Ethernet technology and Internet technology are combined organically to improve the control of computer information resources and show more convenient remote management of computer network. However, some problems still remain to be solved in the current computer network remote control, such as network stability, network security and network protocols. In this regard, we need relevant staff to improve the computer network remote control system, to make it more stable and safe service to the public.

\section{References}

[1] Editorial Department. Network security standards first[J]. Information Technology and Standardization. 2016 (06)

[2] Huahua Liu. Traditional Network and Modern Network Security[J]. Computer Security. 2014 (03)

[3] Lee meter. Research on Network Security Technology Model[J]. Journal of Information and 
Computers (Theory Edition). 2014 (07)

[4] Jing Yan 123. 2016 network security trends[J]. Computer and Network. 2016 (05)

[5] Ye Chunqing. Great concept of network security development[J]. Financial Technology era. 2017 (11)

[6] Shang Dan. New highlights of National Cyber Security Week 2017[J]. Information Engineering. 2017 (10)

[7] "Network Security Summit" held[J]. China Information Security. 2017 (10) 